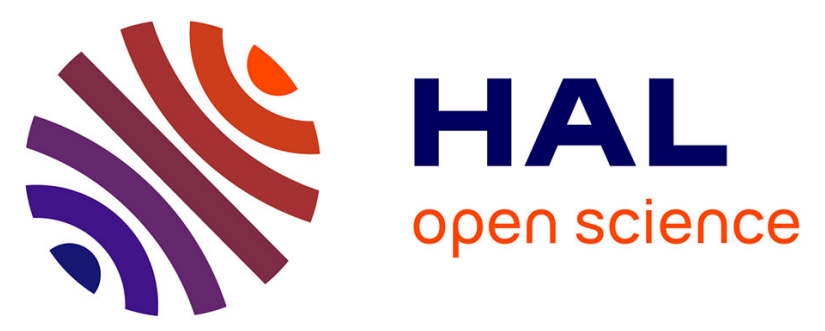

\title{
Intérêt de la musicothérapie dans la prise en charge de la lombalgie chronique en milieu hospitalier (Étude contrôlée, randomisée sur 65 patients)
}

S. Guétin, E. Coudeyre, Marie-Christine Picot, P. Ginies, B. Graber-Duvernay, D. Ratsimba, W. Vanbiervliebt, Jean-Pierre Blayac, Christian Hérisson

\section{To cite this version:}

S. Guétin, E. Coudeyre, Marie-Christine Picot, P. Ginies, B. Graber-Duvernay, et al.. Intérêt de la musicothérapie dans la prise en charge de la lombalgie chronique en milieu hospitalier (Étude contrôlée, randomisée sur 65 patients). Annales de Réadaptation et de Médecine Physique, 2005, 48 (5), pp.217-224. 10.1016/j.annrmp.2005.02.003 . hal-03061617

\section{HAL Id: hal-03061617 \\ https://hal.science/hal-03061617}

Submitted on 25 Oct 2021

HAL is a multi-disciplinary open access archive for the deposit and dissemination of scientific research documents, whether they are published or not. The documents may come from teaching and research institutions in France or abroad, or from public or private research centers.
L'archive ouverte pluridisciplinaire HAL, est destinée au dépôt et à la diffusion de documents scientifiques de niveau recherche, publiés ou non, émanant des établissements d'enseignement et de recherche français ou étrangers, des laboratoires publics ou privés.

\section{(ㄷ)(1) $\$$}

Distributed under a Creative Commons Attribution - NonCommerciall 4.0 International 


\section{Intérêt de la musicothérapie dans les troubles du comportement chez des patients déments hospitalisés}

The interest of music therapy in behavioral disorders among demented hospitalized patients

Rebiha Brahmi, Soraya Ouameri, Jicem Toumi, Enzo Boulardin, Christophe Trivalle

Brahmi R, Praticien attaché associé, rebiha.brahmi@yahoo.fr

Ouameri S, Assistant spécialiste attaché, soraya.tiranime@aphp.fr

Toumi J, Praticien attaché associé, jicem.toumi@aphp.fr

Boulardin E, Musicothérapeute, enzo.boualrdin@gmail.com

Trivalle C, Praticien hospitalier, Chef de service, christophe.trivalle@aphp.fr

Service de Gérontologie 1 et SSR Alzheimer, Hôpital Universitaire Paul Brousse, AP-HP HUPS, 12 avenue Paul vaillant Couturier, 94800 Villejuif.

Correspondance : Dr Trivalle, christophe.trivalle@ aphp.fr 


\section{Résumé}

$\mathrm{Au}$ cours de la maladie d'Alzheimer et des pathologies apparentées, les troubles du comportement jugés perturbateurs représentent une vraie difficulté de prise en charge. Les effets indésirables des psychotropes limitent leur prescription, d'où la nécessité de promouvoir des techniques de soins non médicamenteuses. Dans ce cadre, nous avons réalisé une étude pour mesurer l'impact de la musicothérapie sur la prescription de psychotropes. Cette étude sur 6 mois a inclus 40 patients hospitalisés dans les unités Alzheimer de l'hôpital Universitaire Paul Brousse (AP-HP) et ayant des troubles du comportement jugés perturbateurs. Le programme de musicothérapie prévoyait, pour chaque patient, 3 séances par semaine d'une durée de 15 à 45 minutes. La moyenne d'âge des patients était de 82,9 ans. La maladie d'Alzheimer était le type de démence le plus fréquent (52,5\%). Soixante-cinq pour cent avaient une pathologie très évoluée avec un MMS non testable. Les benzodiazépines (BZD) représentaient $95 \%$ des psychotropes prescrits. Après le programme de musicothérapie, nous avons constaté une baisse de la consommation des benzodiazépines très significative $(\mathrm{p}=0,0002)$ et une tendance à la diminution du recours aux traitements en «si besoin ». Il n'y avait pas de différence dans la prescription des neuroleptiques, des antidépresseurs et des hypnotiques. La prise en charge des troubles du comportement dans la maladie d'Alzheimer doit passer par la limitation des prescriptions de psychotropes et par la promotion des techniques de soins non médicamenteuses comme la musicothérapie.

Mots-clés : maladie d'Alzheimer; troubles du comportement; hospitalisation ; musicothérapie ; psychotropes ; benzodiazépines ; SCPD

\section{Summary}

In the course of Alzheimer's disease and related pathologies, behavioral disorders considered to be disruptive lead to real difficulties for management. The adverse effects of psychotropic 
drugs limit their prescription, hence the need to promote non-drug care techniques. In this context, we conducted a study to measure the impact of music therapy on the prescription of psychotropic drugs. This 6-month study included 40 hospitalized patients in the Alzheimer's units of Paul Brousse University Hospital (AP-HP) with behavioral disorders deemed disruptive. For each patient, the music therapy program provided for 3 sessions per week lasting 15 to 45 minutes. The average age of the patients was 82.9 years. Alzheimer's disease was the most common type of dementia (52.5\%). Sixty-five percent had a very advanced pathology with a non-testable MMS. Benzodiazepines (BZDs) accounted for $95 \%$ of the psychotropic drugs prescribed. Following the music therapy program, we found a very significant decrease in benzodiazepine consumption $(\mathrm{p}=0.0002)$ and a decrease in the use of "if needed" treatments. There was no difference in the prescriptions of neuroleptics, antidepressants or hypnotics. The management of behavioral disorders in Alzheimer's disease should include the restriction of psychotropic prescriptions and the promotion of non-medical care techniques, such as music therapy.

Keywords : Alzheimer's disease ; behavioral disorders; hospitalization; music therapy; psychotropic drugs; benzodiazepines; BPSD 
Selon la Haute autorité de santé (HAS) [1], les symptômes comportementaux et psychologiques de la démence (SCPD) sont classés en deux catégories selon qu'il s'agit de comportements perturbateurs ou non perturbateurs. Parmi les comportements perturbateurs figurent : délire, hallucinations, hyperactivité, agitation/agressivité, euphorie, désinhibition, irritabilité/instabilité de l'humeur et comportement moteur aberrant (déambulation). Parmi les comportements non perturbateurs figurent: dépression, anxiété, apathie, symptômes végétatifs, troubles du sommeil et troubles de l'appétit.

Dans les maladies d'Alzheimer et apparentées, face aux troubles du comportement perturbateurs on a souvent recours aux psychotropes et en particulier aux neuroleptiques, générateurs d'effets secondaires importants : troubles de la marche, chutes, agitation, confusion, ralentissement psychomoteur... D'où l'intérêt suscité, depuis quelques années, par les thérapeutiques non médicamenteuses.

Parmi les thérapeutiques non médicamenteuses figure la musicothérapie, une forme particulière d'art-thérapie, qui utilise la musique comme un moyen de soulager ou d'apaiser la douleur, mais aussi les troubles du comportement. Ainsi, plusieurs études et méta-analyses [2-6] se sont intéressées aux effets de la musicothérapie dans la prise en charge des troubles du comportement des sujets âgés et en particulier ceux atteints de la maladie d'Alzheimer.

C'est dans ce cadre que nous nous sommes proposé de réaliser une étude pour mesurer l'impact de la musicothérapie sur la prescription de psychotropes.

\section{Population et méthodes}

\section{Les patients}


Il s'agit d'une étude descriptive transversale qui s'est déroulée pendant 6 mois (de novembre 2017 jusqu'au mois d'avril 2018) dans les unités de soins de suite et réadaptation (SSR, 52 patients) et de soins de longue durée (SLD, 71 patients) à orientation Alzheimer de l'hôpital universitaire Paul Brousse de Villejuif (AP-HP).

Pouvaient participer tous les patients âgés hospitalisés en SSR et SLD Alzheimer durant la période de l'étude et présentant une maladie d'Alzheimer ou une pathologie apparentée et des troubles de comportement jugés perturbateurs par les équipes soignantes et les médecins.

Ont été exclus les patients dont la famille, la personne de confiance ou le tuteur ont refusé qu'ils participent à cette activité ; ceux qui ont refusé l'entretien initial, et ceux sans troubles du comportement ou avec des troubles du comportement non productifs.

\section{Principe de la musicothérapie}

La musicothérapie est dite « réceptive » lorsqu'elle propose, au sein d'une relation thérapeutique, des dispositifs fondés sur l'écoute, faisant appel à une association libre et une élaboration psychique. Elle s'appuie aussi sur les effets psychoaffectifs et psychophysiologiques de la musique, mis en évidence actuellement par des travaux en neuropsychologie et neurobiologie [7]. La musicothérapie est dite « active » lorsqu'elle propose des dispositifs de travail thérapeutique privilégiant la production sonore et musicale, l'improvisation, la créativité. Les éléments musicaux (rythme, son, timbre, intensité) sont utilisés afin de permettre à la personne de s'exprimer, communiquer, créer des liens tout en accomplissant un travail de structuration identitaire. Ces deux formes de musicothérapie se pratiquent en séances de groupe ou en séances individuelles et ont chacune leurs spécificités dans les deux types de séances. 
La prise en charge individuelle permet des améliorations sur les plans psychologique, cognitif et comportemental. Elle est avant tout un moyen de s'exprimer. Elle permet de stimuler la créativité du patient en proposant des directions de travail variées à chaque séance, telles que l'improvisation, la création d'une ambiance, ou encore la transposition, musicalement, d'un sentiment, d'un état. Le patient retrouve une certaine autonomie en s'exprimant comme il le veut, en choisissant le ou les instruments qu'il utilisera pour jouer, ou même tout simplement dans la façon de communiquer.

Quant à la prise en charge en groupe, il s'agit de proposer l'audition d'œuvres préalablement choisies par le musicothérapeute. La séance se compose de la suite de plusieurs associations de courts extraits sélectionnés pour contraster sur un paramètre musical, psychologique, ou encore physique: par exemple, une musique rythmique et une musique mélodique, un mouvement lent et un mouvement rapide, une musique vocale et une musique orchestrale. La comparaison entre les deux écoutes facilite un mouvement de différenciation, de prise de conscience, et le positionnement du patient par rapport à une préférence musicale.

\section{Rôle du musicothérapeute}

Le musicothérapeute clinicien est un professionnel qui exerce une pratique soignante. Il vise à favoriser l'expression et le mieux-être des patients. Son travail s'effectue dans le champ des thérapies de soutien et de réadaptation. Ses suivis sont individuels ou en petits groupes et peuvent se réaliser au long cours. Il intervient au sein des équipes pluridisciplinaires en collaboration avec les professionnels de santé. Ses secteurs d'intervention sont très variés. La musicothérapie est inscrite au répertoire national des Certifications professionnelles (RNCP), et il existe une Fédération française de musicothérapie (www.musicotherapie-federationfrancaise.com). 
La prise en charge par un musicothérapeute clinicien s'organise de manière très structurée :

- intervention sur prescription médicale, indiquant le diagnostic et le motif de la demande de prise en charge ;

- mise en place d'un cadre thérapeutique : une séance de thérapie se déroule dans un cadre de lieu et de temps, avec un aménagement du son acoustique, des stimuli, de la luminosité afin d'amener le patient à une notion de temps et de repère rassurant dans lequel il va pouvoir évoluer;

- bilan psychomusical à partir d'un entretien avec le patient afin de définir, voire deviner le cas échéant, son profil et ses affinités musicales, complété par un recueil de données auprès de la famille et/ou du personnel soignant, tout en tenant compte de ses troubles neurosensoriels ;

- établir un projet thérapeutique : adapté et personnalisé selon le profil réceptif ou actif, en définissant les objectifs de la prise en charge des troubles du comportement ;

- le bilan : le musicothérapeute évalue subjectivement l'amélioration de l'anxiété et de l'agitation.

Le programme de musicothérapie prévoyait, pour chaque patient, 3 séances par semaine d'une durée de 15 à 45 minutes.

\section{Evaluation}

Les données générales ont été recueillies à l'aide d'un questionnaire standardisé (âge, sexe, pathologies associées, type de démence, troubles du comportement, nombre et types de médicaments...). Une évaluation par le MMS [8] a été faite pour chaque patient inclus dans l'étude.

Une évaluation a été faite de façon subjective par le musicothérapeute lors de chaque séance. 
Pour estimer l'impact de la musicothérapie d'une façon objective, nous avons évalué les différentes thérapeutiques utilisées avant et après la période de prise en charge de 6 mois.

\section{Statistiques}

Pour comparer les prescriptions médicamenteuses avant et après la période de musicothérapie, nous avons utilisé le Test de McNemar pour groupes appariés avec contrôle par le test exact de Fisher. Un p $<0,05$ a été considéré comme significatif.

\section{Résultats}

\section{Données générales}

Sur les 6 mois de l'étude, 40 patients ont pu être inclus. Les principales caractéristiques de notre population figurent sur le tableau 1. La moyenne d'âge des patients était de 82,9 ans. La maladie d'Alzheimer était le type de démence le plus fréquent (52,5\%). Dans $65 \%$ des cas la pathologie était très sévère et non testable (MMS à zéro). Les troubles anxiodépressifs constituaient la majorité des comorbidités psychiatriques avec une fréquence de 32,5\%.

Les pathologies cardiovasculaires, ostéo-articulaires ainsi que le diabète étaient respectivement les comorbidités les plus fréquemment retrouvées dans notre population. La quasi-totalité des patients était polymédiquée (95\%) avec $\geq 7$ médicaments. Les benzodiazépines (BZD) représentaient $95 \%$ des psychotropes consommés par les patients.

\section{Profil neuropsychiatrique des patients}


Tous les troubles du comportement (SCPD) qu'ils soient perturbateurs ou non ont été évalués (tableau 2). Les troubles du comportement essentiellement présents dans notre population étaient la dépression, l'anxiété ainsi que l'agressivité avec des fréquences de 57,5\%. Le risque de fugue concernait $12,5 \%$ des patients.

\section{Type de prise en charge par le musicothérapeute}

Les patients ont bénéficié de séances de prise en charge individuelle et/ou en groupe comme cela est présenté sur le tableau 3. Vingt-quatre $(60 \%)$ ont d'abord eu une prise en charge individuelle puis en groupe.

\section{Evaluation qualitative effectuée par le musicothérapeute}

Au cours de chaque séance (individuelle ou en groupe), le musicothérapeute notait l'impact observé sur chaque patient. L’impact des séances était aussi discuté avec les équipes soignantes. Ses conclusions globales ont été les suivantes :

- Mieux-être instantané à la fin de la séance de musique dès le début de la thérapie ;

- Bien-être après 5 semaines à raison de 3 séances par semaine ;

- Baisse de l'anxiété et de l'agitation dès les premières semaines ;

- Diminution de l'opposition aux soins et de l'agressivité rapportée par les soignants ;

- Pas d'effet constaté sur les troubles cognitifs.

\section{Evolution de la prise en charge médicamenteuse}

Notre étude a montré une baisse de la consommation des benzodiazépines très significative $(\mathrm{p}=0,0002)$ et une tendance à la diminution du recours aux traitements en «si besoin « 
(tableau 4). Il n'y avait pas de différence dans la prescription des neuroleptiques, des antidépresseurs et des hypnotiques.

\section{Discussion}

Notre travail s'est intéressé aux effets de la musique sur les troubles du comportement chez des patients atteints de pathologies cognitives et principalement de la maladie d'Alzheimer. Notre objectif était de montrer l'impact de la musique sur le contrôle des symptômes psychocomportementaux en mesurant la baisse potentielle des traitements psychotropes.

La musique est un vecteur émotionnel puissant qui impacte fortement la récupération en mémoire. La perte des souvenirs s'accompagnant d'une perte d'identité et d'estime de soi peut provoquer des troubles du comportement. D'où l'intérêt potentiel de la musicothérapie.

Un premier résultat est que nous avons pu montrer qu'il était possible de mettre en place un programme de 6 mois de musicothérapie (à raison de 3 séances par semaine) à l'hôpital en SSR et en SLD pour des malades ayant une pathologie cognitive (maladie d'Alzheimer dans $52 \%$ des cas), souvent très évoluée (65\% non testables au MMS) et avec des troubles du comportement.

D'un point de vue subjectif, la musique semble permettre une meilleure récupération des états affectifs et émotionnels. Dans notre étude, il était rapporté par le musicothérapeute une amélioration du bien-être, une baisse de l'anxiété et de l'agitation dès les premières semaines et une diminution de l'opposition aux soins et de l'agressivité rapportée par les soignants. Pour Tsoi et al [9], la musicothérapie réceptive réduirait l'agitation, l'anxiété et les SCPD chez les personnes âgées atteintes de démence et aurait même des effets et des résultats meilleurs que la thérapie active. De plus, elle est plus facilement réalisable et moins coûteuse. 
Dans la littérature, des effets positifs ont pu être observés sur la dépression, les interactions sociales, les troubles de comportement (agitation, agressivité, nervosité), la désorientation temporo-spatiale ainsi que sur la capacité à rester engagé dans une activité présente et à planifier une activité future [10]. Il a aussi été montré un effet bénéfique d'ateliers musicaux sur l'état émotionnel de patients atteints de démence avec une diminution des troubles du comportement et une réduction du fardeau ressenti par l'aidant [11]. Cependant, pour Scott et Kidd [12] les preuves étaient insuffisantes pour conclure à un effet de la musicothérapie sur l'anxiété, la dépression et l'agitation chez des personnes âgées démentes en institutions.

Notre programme de musicothérapie a duré 6 mois. Il semble que la durée de prise en charge soit un élément important à évaluer. Ainsi, Ueda et al [13] qui ont montré que la musicothérapie avait un effet modéré sur l'anxiété et un effet léger sur les autres troubles du comportement, suggèrent que plus la durée de la musicothérapie est longue (supérieure à 3 mois), meilleurs seront les résultats sur les troubles de comportement. De même, Li et al [14] concluent dans une méta-analyse que la musicothérapie sur une courte durée n'avait pas d'effets sur les troubles cognitifs.

En ce qui concerne les fonctions cognitives, ce que nous n'avons pas évalué dans notre étude, Moussard et al [11] ont étudié l'apprentissage de la parole chantée versus de la parole prononcée, dans un groupe de sujets sains et un groupe de patients atteints de maladie d'Alzheimer. Les résultats ont montré une meilleure rétention à long terme des paroles chantées, après 4 semaines et ce, dans les 2 groupes. Palisson et al [15] ont obtenu des résultats similaires dans leur étude et ont conclu qu'associer une musique pendant l'encodage facilitait l'apprentissage et la rétention chez les sujets atteints de la maladie d'Alzheimer. Une étude randomisée (musicothérapie versus activité de cuisine deux fois une heure par semaine pendant 4 semaines) [16] chez 48 patients déments n'a montré aucun bénéfice sur les 
fonctions cognitives. Par contre, les deux activités amélioraient le bien-être émotionnel et diminuaient les troubles du comportement chez les patients et le stress chez les aidants.

De nombreux travaux indiquent en cas de lésion cérébrale, que la sollicitation des aires cérébrales impliquées dans le traitement de la musique avait un effet positif sur les capacités cognitives (attention, mémoire et langage) et favorisaient la plasticité cérébrale.

Selon Louart [17], la répétition de stimuli musicaux contribue à favoriser les échanges d'informations entre les deux hémisphères et à augmenter le nombre de neurones qui assurent cette communication, ce qui a pour effet de modifier la structure du cerveau. Chez les musiciens, ces modifications se traduisent par des changements visibles, sur le plan anatomique avec, par exemple, une plus forte densité du corps calleux par rapport aux non musiciens.

L'ouvrage coordonné par Platel et Thomas-Antérion [18] souligne également le rôle privilégié de la musique dans la médiation et la réhabilitation des troubles cognitifs et psycho-comportementaux, en particulier dans la maladie d'Alzheimer, pour la mémoire implicite, le langage, la plasticité cérébrale.

L'étude de El Haj et al [19] témoigne de l'effet bénéfique de la musique sur la récupération de la mémoire dans la maladie d'Alzheimer avec une réduction de l'anxiété et des troubles du comportement. Il semblerait, de plus, qu'utiliser la musique sollicite moins les mécanismes de contrôle cognitif, mécanismes déficitaires chez ces patients. En effet, la musique semble permettre un fonctionnement cognitif plus automatique/involontaire faisant davantage intervenir les mécanismes émotionnels. Ces mécanismes sont plus longtemps préservés dans la maladie d'Alzheimer et pourraient ainsi être davantage sollicités dans la récupération des souvenirs. 
Spielberger et al [20] ont mis en évidence une amélioration considérable du rappel des souvenirs dans la condition musicale, avec de plus, une réduction significative de l'anxiété ainsi que des troubles attentionnels et exécutifs, en favorisant l'émergence d'un contexte émotionnel agréable. Parmi les patients de notre étude, 32,5\% avaient des troubles anxiodépressifs.

Dans notre étude, nous avons montré une tendance à la baisse de prescription de psychotropes en «si besoin» et une diminution significative de l'utilisation des benzodiazépines. Dans une étude de plus grande envergure, Thomas et al [21], sur 12905 résidents dans 98 maisons de retraite aux Etats-Unis en 2013 - ils comparaient les symptômes comportementaux et psychologiques associés à la démence avant et après l'introduction d'un programme individualisé de musique - ont constaté une diminution de la consommation des antipsychotiques qui atteignait $20 \%$ par rapport au groupe témoin.

La musique a ainsi toute sa place dans la prise en charge en remédiation cognitive y compris chez les patients qui présentent une détérioration cognitive avancée. Elle semble améliorer le bien-être, diminuer l'anxiété et les troubles du comportement et entraîner une diminution des prescriptions de psychotropes et en particulier de benzodiazépines.

\section{Limites de l'étude}

On peut noter un manque de puissance $(n=40)$ et une étude limitée dans le temps $(6$ mois $)$. Les troubles du comportement n'étaient pas homogènes, de même que les causes de démence. Une seule évaluation objective a été faite : la consommation médicamenteuse avant et après la musicothérapie. Ces facteurs ont pu limiter l'évaluation de l'impact de la musicothérapie dans notre population.

De même, le fait que les patients avaient majoritairement une démence très sévère (MMS non testable) peut avoir diminué la réceptivité à la musicothérapie. 
Il aurait été intéressant de faire une évaluation des SCPD avec l'échelle NPI (Inventaire Neuropsychiatrique) avant et après. Ce qui sera fait lors d'une prochaine évaluation.

Il a aussi été difficile de connaître l'affinité ou la pratique musicale de tous les patients, ce qui aurait pu être utile pour personnaliser la prise en charge et obtenir de meilleurs résultats. En effet, les patients ayant déjà eu une pratique musicale par le passé sont plus réceptifs à la musicothérapie.

Comme la pratique de la musicothérapie n'existait pas auparavant en SSR et en SLD, le musicothérapeute a dû créer, construire et développer, à son arrivée, un projet spécifique : planning, détermination d'un lieu géographique de travail, fiche de musicothérapie, programme adapté, sélection des patients. Ceci a limité les possibilités d'évaluation (NPI, MMS ou autres échelles avant/après) du fait d'un manque de temps.

De plus, il a fallu instaurer une relation de confiance avec les équipes pluridisciplinaires et avec les patients au fil des séances, condition incontournable au bon déroulement des prises en soin. De ce fait, une durée supérieure à 6 mois aurait probablement été nécessaire.

\section{Conclusion}

Les comportements perturbateurs sont d'autant plus difficiles à corriger qu'ils sont anciens. Les recommandations de la HAS [1] sont faites pour diminuer la prescription de psychotropes et de contentions. Pour cela il faut développer les thérapies non médicamenteuses, parmi lesquelles figure la musicothérapie. Cette prise en charge par la musicothérapie nécessite plusieurs séances par semaine s'étalant sur au moins deux mois.

Notre travail a permis de montrer qu'il était possible de mettre en place des séances de musicothérapies à l'hôpital (3 fois par semaine pendant 6 mois), en SSR comme en SLD, y compris pour des patients ayant une pathologie démentielle déjà très avancée. Malgré les limites de notre étude, et en dehors d'une amélioration subjective évaluée par le 
musicothérapeute, nous avons mis en évidence une diminution de prescriptions des benzodiazépines.

Des études avec plus de patients, une utilisation de la NPI et une durée plus prolongée sont nécessaires pour confirmer et développer nos résultats.

Conflits d'intérêts : aucun. 


\section{Références}

1. HAS. Maladie d'Alzheimer et maladies apparentées: prise en charge des troubles du comportement perturbateurs. Recommandations de bonne pratique, mai 2009. https://www.has-sante.fr/portail/upload/docs/application/pdf/2009-07/maladie_dalzheimertroubles_du_comportement_perturbateurs-recommandations.pdf Consulté le 4 janvier 2019.

2. Ing-Randolph AR, Phillips LR, Williams AB. Group music interventions for dementiaassociated anxiety: A systematic review. Int J Nurs Stud 2015;52(11):1775-84.

3. Chevreau P, Nizard I, Allain P. Récupérer ses souvenirs grâce à la musique dans la maladie d'Alzheimer. Geriatr Psychol Neuropsychiatr Vieil 2017 ; 15(3) : 309-18.

4. Zhang Y, Cai J, An L, et al. Does music therapy enhance behavioral and cognitive function in elderly dementia patients? A systematic review and meta-analysis. Ageing Res Rev 2017; 35: $1-11$.

5. Gómez-Romero M. Benefits of music therapy on behaviour disorders in subjects diagnosed with dementia: A systematic review. Neurología 2017;32(4):253-63.

6. Gómez Gallego M, Gómez García J. Music therapy and Alzheimer's disease: Cognitive, psychological, and behavioural effects. Neurología 2017;32(5):300-8.

7. Platel H, Groussard M. La mémoire sémantique musicale: apport des données de la neuropsychologie clinique et de la neuro-imagerie fonctionnelle. Rev Neuropsychol $2010 ; 2$ (1) : 61-9. 
8. Creavin ST, Wisniewski S, Noel-Storr AH, et al. Mini-Mental State Examination (MMSE) for the detection of dementia in clinically unevaluated people aged 65 and over in community and primary care populations. Cochrane Database Syst Rev 2016;(1):CD011145.

9. Tsoi KKF, Chan JYC, Ng YM, et al. Receptive music therapy is more effective than interactive music therapy to relieve behavioral and psychological symptoms of dementia: A systematic review and meta-analysis. J Am Med Dir Assoc 2018;19(7):568-576.e3.

10. van der Steen JT, Smaling HJ, van der Wouden JC, et al. Music-based therapeutic interventions for people with dementia. Cochrane Database Syst Rev 2018;7:CD003477.

11. Moussard A, Bigand E, Belleville S, et al. Learning sung lyrics aids retention in normal ageing and Alzheimer's disease. Neuropsychol Rehabil 2014 ; 24 : 1-24.

12. Scott SCE, Kidd AC. A scoping review of music and anxiety, depression and agitation in older people with dementia in residential facilities and specialist care units. European Geriatric Medicine 2016; 7: 488-91.

13. Ueda T, Suzukamo Y, Sato M, et al. Effects of music therapy on behavioral and psychological symptoms of dementia: A systematic review and meta-analysis. Ageing Res Rev 2013;12(2):628-41. 
14. Li HC, Wang HH, Chou FH, et al. The effect of music therapy on cognitive functioning among older adults: A systematic review and meta-analysis. J Am Med Dir Assoc 2015;16(1):71-7.

15. Palisson J, Roussel-Baclet C, Maillet D, et al. Music enhances verbal episodic memory in Alzheimer's disease. J Clin Exp Neuropsychol 2015 ; 37 : 503-17.

16. Narme P, Clément S, Ehrlé N, et al. Efficacy of musical interventions in dementia: evidence from a randomized controlled trial. J Alzheimers Dis 2014; 38 : 359-69.

17. Louart C. La musique pour soigner la mémoire, septembre 2016. https://lejournal.cnrs.fr/articles/la-musique-pour-soigner-la-memoire Consulté le 4 janvier 2019.

18. Platel H, Thomas-Antérion C. Neuropsychologie et art. Belgique: DeBoeck Solal, 2014.

19. El Haj M, Antoine P, Nandrino JL, et al. Autobiographical memory decline in Alzheimer's disease, a theoretical and clinical overview. Ageing Res Rev 2015; 23: 183-92.

20. Spielberger CD, Gorsuch RL, Lushene RE. The state trait anxiety inventory. Mountain View: Consulting psychologists Press, 1970.

21. Thomas KS, Baier R, Kosar C, et al. Individualized music program is associated with improved outcome for US nursing home residents with dementia. Am J Geriatr Psychiatry $2017 ; 25: 931-8$. 
Tableau 1 : Principales caractéristiques des 40 patients inclus dans l'étude.

\begin{tabular}{|c|c|c|c|}
\hline Caractéristique & & $\begin{array}{l}\text { Nou } \\
\text { moyenne }\end{array}$ & $\%$ \\
\hline Sexe & $\begin{array}{l}\text { Hommes } \\
\text { Femmes }\end{array}$ & $\begin{array}{l}19 \\
21\end{array}$ & $\begin{array}{l}47,5 \% \\
52,5 \%\end{array}$ \\
\hline Moyenne d'âge (années) & $\begin{array}{l}\text { Hommes } \\
\text { Femmes }\end{array}$ & $\begin{array}{l}82,9 \\
82 \\
83,4\end{array}$ & \\
\hline Type de démence & $\begin{array}{l}\text { Alzheimer } \\
\text { Mixtes } \\
\text { Corps de Lewy } \\
\text { Parkinson } \\
\text { Korsakov } \\
\text { non étiqueté }\end{array}$ & $\begin{array}{l}21 \\
12 \\
2 \\
1 \\
1 \\
3\end{array}$ & $\begin{array}{l}52,5 \% \\
30 \% \\
5 \% \\
2,5 \% \\
2,5 \% \\
7,5 \%\end{array}$ \\
\hline MMS & $\begin{array}{l}15-20 \\
10-14 \\
<10 \\
\text { non évaluables }(=0)\end{array}$ & $\begin{array}{l}4 \\
5 \\
5 \\
26\end{array}$ & $\begin{array}{l}10 \% \\
12,5 \% \\
12,5 \% \\
65 \%\end{array}$ \\
\hline $\begin{array}{l}\text { Comorbidités } \\
\qquad \begin{array}{l}\text { - psychiatriques }\end{array}\end{array}$ & $\begin{array}{l}\text { Psychose hallucinatoire } \\
\text { chronique } \\
\text { Anxiodépressive }\end{array}$ & $\begin{array}{l}6 \\
13 \\
5\end{array}$ & $\begin{array}{l}15 \% \\
32,5 \% \\
12,5 \%\end{array}$ \\
\hline
\end{tabular}




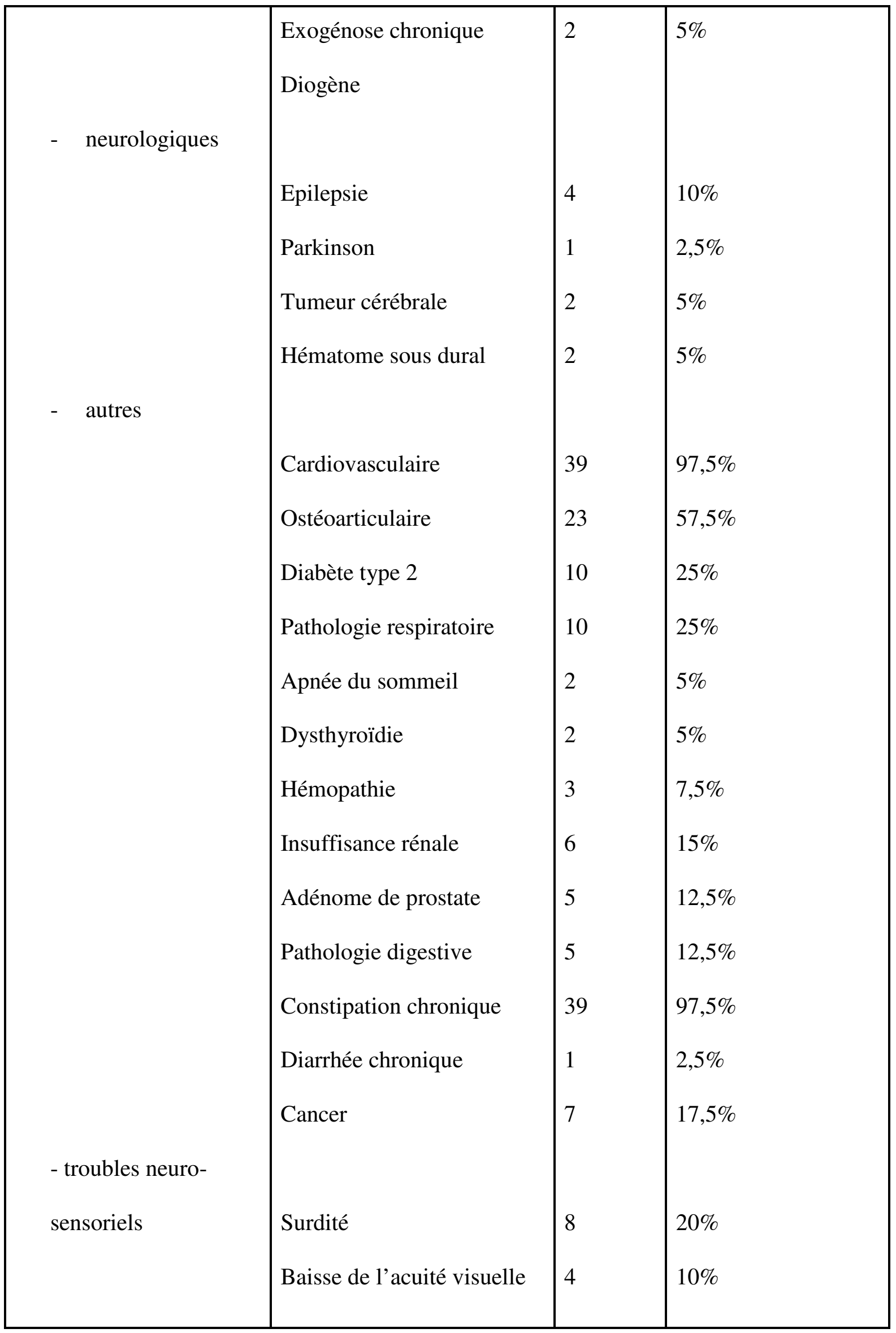




\begin{tabular}{|l|l|l|l|}
\hline Nombre de médicaments & $<7$ médicaments & 2 & $5 \%$ \\
& $\geq 7$ médicaments & 38 & $95 \%$ \\
\hline Psychotropes & Benzodiazépines & 38 & $95 \%$ \\
& Antidépresseurs & 20 & $50 \%$ \\
& Neuroleptiques & 22 & $52,5 \%$ \\
\hline
\end{tabular}

MMS : Mini Mental Status. 
Tableau 2 : Troubles du comportement (SCPD) des 40 patients.

\begin{tabular}{|l|l|l|}
\hline Trouble du comportement & $\mathbf{n}$ & \% \\
\hline Délire & 14 & $35 \%$ \\
\hline Hallucination & 20 & $50 \%$ \\
\hline Agitation & 20 & $50 \%$ \\
\hline Agressivité & 23 & $57,5 \%$ \\
\hline Désinhibition & 6 & $15 \%$ \\
\hline Anxiété & 23 & $57,5 \%$ \\
\hline Comportement moteur aberrant & 16 & $40 \%$ \\
\hline Dépression & 23 & $57,5 \%$ \\
\hline Apathie & 10 & $17,5 \%$ \\
\hline Troubles sommeil & 5 & $32,5 \%$ \\
\hline Opposition aux soins & 13 & $25 \%$ \\
\hline Fugues & $7,5 \%$ \\
\hline
\end{tabular}


Tableau 3 : Type de prise en charge (PEC) en musicothérapie.

\begin{tabular}{|l|l|l|}
\hline Type & $\mathbf{n}$ & $\%$ \\
\hline PEC individuelle & 8 & $20 \%$ \\
\hline PEC en groupe & 8 & $20 \%$ \\
\hline D'abord individuelle puis en & 24 & $60 \%$ \\
\hline groupe & & \\
\hline
\end{tabular}


Tableau 4: Comparaison des traitements pris avant la prise en charge par musicothérapie et après.

\begin{tabular}{|l|l|l|l|}
\hline Psychotropes & Avant & Après & p \\
\hline Neuroleptiques & 22 & 22 & NS \\
\hline Antidépresseurs & 20 & 20 & NS \\
\hline Benzodiazépines & 38 & 17 & 0,0002 \\
\hline Psychotropes en « si besoin » & 30 & 21 & 0,07 \\
\hline Hypnotiques & 13 & 10 & NS \\
\hline
\end{tabular}

NS = Non significatif. 